\title{
Totally laparoscopic complete bursectomy and D2 lymphadenectomy in radical total gastrectomy: an outside bursa omentalis approach
}

\author{
Liaonan Zou ${ }^{1}$ Wenjun Xiong ${ }^{1} \cdot$ Delong $\mathrm{Mo}^{2} \cdot$ Guobin Chen $^{1} \cdot{\text { Yaobin } \mathrm{He}^{1}}^{1}$ \\ Hongming $\mathrm{Li}^{1} \cdot$ Ping Tan ${ }^{1} \cdot$ Wei Wang ${ }^{1} \cdot J^{\prime}$ Wan $^{1}$
}

Received: 19 August 2015/Accepted: 18 November 2015/Published online: 16 December 2015

(C) Springer Science+Business Media New York 2015

\begin{abstract}
Background Bursectomy is regarded as a standard surgical procedure during gastrectomy for serosa-positive gastric cancer in Japanese gastric cancer treatment guidelines (Japanese Gastric Cancer Association in Gastric Cancer 14:113-123, 2011). As a consequence, bursectomy is widely performed in open gastrectomy. However, laparoscopic gastrectomy with bursectomy is rare. Based on our previous experience of laparoscopic bursectomy in distal gastrectomy (Zou et al. in Oncol Lett 10:99-102, 2015), herein, we described the technique of totally laparoscopic radical total gastrectomy with complete bursectomy using an outside bursa omentalis approach.

Methods Firstly, the transverse mesocolon and distal gastric membrane were separated from right to left, and the right gastroepiploica vessels were ligated at root with No. 6 lymph nodes (LNs) dissection followed by the pancreas membrane dissection from pancreas head to pancreas tail. Secondly, the anterior plane of transverse mesocolon was dissected from left to right starting from the lower pole of spleen, and the membrane of pancreas tail was separated to combine the pancreas anterior plane with No. 4s, 10, 11d and 2 LNs dissection. Thirdly, the lesser omental
\end{abstract}

Electronic supplementary material The online version of this article (doi:10.1007/s00464-015-4702-z) contains supplementary material, which is available to authorized users.

Jin Wan

gdphtcmwanjin@163.com

1 Department of Gastrointestinal (Tumor) Surgery, Guangdong Province Hospital of Chinese Medicine, The Second Affiliated Hospital of Guangzhou University of Chinese Medicine, Dade Road No. 111, Guangzhou 510512, China

2 Department of General Surgery, Hospital of Traditional Chinese Medicine of Zhongshan, Kangxin Road No. 3, Zhongshan, China was dissected from right to left with No. 5 and 12a LNs dissection, and the duodenum was transected. Then, the No. 7, 8, 9 and 11p LNs were dissected followed by No. 1 LNs dissection. Finally, a Roux-en-Y esophagojejunostomy was carried out intracorporeally with a linear cutter.

Results Thirty-two patients with advanced proximal gastric cancer underwent laparoscopic total gastrectomy with complete bursectomy using an approach outside bursa omentalis. One bowel obstruction and one pulmonary infection were recorded and cured with conservative measure. The mean operative time was $253.3 \pm 31.3 \mathrm{~min}$ with a mean blood loss of $90.5 \pm 23.1 \mathrm{ml}$. The mean length of stay was $10.6 \pm 2.6$ days.

Conclusion Laparoscopic radical total gastrectomy with complete bursectomy using an outside bursa omentalis approach is feasible and safe in experienced hands with favorable short outcome. Further studies were needed for its advanced application.

Keywords Laparoscopic surgery $\cdot$ Radical total gastrectomy $\cdot$ Complete bursectomy $\cdot$ Outside bursa omentalis approach

Compliance with ethical standards

Disclosures Liaonan Zou, Wenjun Xiong, Delong Mo, Guobin Chen, Yaobin He, Hongming Li, Ping Tan, Wei Wang and Jin Wan have no conflicts of interest or financial ties to disclose.

\section{References}

1. Japanese Gastric Cancer Association (2011) Japanese gastric cancer treatment guidelines 2010 (ver. 3). Gastric Cancer 14:113-123

2. Zou LN, He YB, Li HM, Diao DC, Mo DL, Wang W, Wan J (2015) Surgical skills for laparoscopic resection of the bursa omentalis and lymph node scavenging with radical gastrectomy. Oncol Lett 10:99-102 